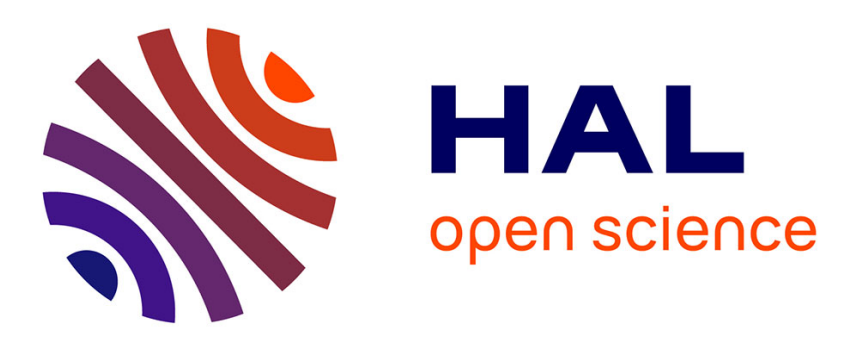

\title{
An Autoethnographic Study of HCI Effort Estimation in Outsourced Software Development
}

Shalaka Dighe, Anirudha Joshi

\section{To cite this version:}

Shalaka Dighe, Anirudha Joshi. An Autoethnographic Study of HCI Effort Estimation in Outsourced Software Development. 5th International Conference on Human-Centred Software Engineering (HCSE), Sep 2014, Paderborn, Germany. pp.19-35, 10.1007/978-3-662-44811-3_2 . hal01405062

\section{HAL Id: hal-01405062 \\ https://hal.inria.fr/hal-01405062}

Submitted on 29 Nov 2016

HAL is a multi-disciplinary open access archive for the deposit and dissemination of scientific research documents, whether they are published or not. The documents may come from teaching and research institutions in France or abroad, or from public or private research centers.
L'archive ouverte pluridisciplinaire HAL, est destinée au dépôt et à la diffusion de documents scientifiques de niveau recherche, publiés ou non, émanant des établissements d'enseignement et de recherche français ou étrangers, des laboratoires publics ou privés. 


\title{
An Autoethnographic Study of HCI Effort Estimation in Outsourced Software Development
}

\author{
Shalaka Dighe ${ }^{1}$, Anirudha Joshi ${ }^{2}$ \\ ${ }^{1}$ Tech Mahindra Ltd., India \\ shalakade techmahindra.com \\ 2 IDC, IIT Bombay, India \\ anirudha@itb.ac.in
}

\begin{abstract}
A fair amount of literature has been published concerning the gaps between HCI and software engineering. However, most of it tends to look at the effects of these gaps rather than their causes. We argue that the use of autoethnographic methods would help us in identifying the root causes of these gaps and can bring us closer to finding potential solutions. In this paper, we focus on issues associated with effort estimation for HCI activities in three projects in three typical engagement models for outsourced software development projects in a mainstream IT company in India, namely Fixed Price model, Mixed model, and Time \& Material model. We found that the HCI practitioner needs to negotiate her position with several members of the team, both within the vendor and client organisations. At times, a foot-in-the-door project turns out to be a footin-the-mouth project. At other times, it leads to inefficiencies and imbalance of work load. The autoethnographic approach led to reflexive thinking by the HCI practitioner, helping her to develop a deeper understanding of all aspects of a problem, and bringing her closer to potential solutions in some cases. The paper also brings to light several aspects of autoethnography as a method, which can influence effort estimation of $\mathrm{HCI}$ activities for future projects.
\end{abstract}

\section{$1 \quad$ Introduction}

It has been more than three decades since Human Computer Interaction (HCI) has been recognized as an independent field of study. HCI has since then grown into a compelling discipline that asserts due consideration in the development of software. Software Engineering (SE) also recognizes the relevance of HCI methods in SE processes in their research. Nevertheless, there are major gaps in integrating HCI and SE in both theory and practice [1]. A review of SE literature reveals that there is still a lot to be desired with regards to references of HCI activities in SE processes, and both fields appear to be severely disjointed especially in the initial phases in the software development process [2] [3].

In practice, there is a substantial lack of mutual understanding among software engineers (SEs) and HCI practitioners, and research testimonies from their respective fields do not appear to strongly influence this interaction. The distance between the disciplines is perhaps the largest in context of outsourced software development pro- 
jects; particularly if the teams are distributed and there are greater gaps of culture, time and distance between team members.

This research aims to identify issues related to entrenchment of HCI activities in SE practices in Indian organisations and their overseas counterparts wherever possible. The Indian Information Technology (IT) sector was born in 1968 and has seen an exponential growth to over 2.8 million people employed in this sector by 2012 [4]. It has also evolved from the initial era of staff augmentation services (also known as 'body shopping') to the current state of specialized software engineering and IT consulting. Most organisations work in an 'onsite-offshore' engagement model with the customers, where the 'onsite' employees, who are collocated with the customer, bring in a synergy with stakeholders, whereas the 'offshore' employees working from India help in building a cost advantage. Clearly, we are looking at a 'culture' that resides within these organisations, with the members of this culture sharing distinct experiences, behaviours, and even language.

Qualitative research with SEs and HCI practitioners to identify gaps in practice has been done though surveys [5] [6], but the root causes for the gaps seem to remain elusive. Perhaps these causes are rooted somewhere in the practical and sociological aspects of software development in practice, in the day-to-day interactions between people from different disciplines, regions and organisations, the process of their decision making, and their power structures. Perhaps surveys do not capture these intricacies sufficiently. Rönkkö has successfully used ethnographic approach to identify challenges in applying HCI methods in several SE projects, but the studies are either limited to singular projects, or in academic projects outside the environment of software organisations [7] [8].

Our research takes an autoethnographic approach to understand how software development works in practice; how small actions from day to day life have an unanticipated impact on a project in the longer term, how decisions get made, what role do the HCI practitioners, SEs and customers play, and what are the root causes that impede the integration of HCI activities in the development of software. We base our work in the context of mainstream IT industry in offshore software development.

In section 2 of this paper, we first summarize prior work done in the area of HCI-SE integration, autoethnography, and HCI effort estimation. Section 3 describes our study with respect to the research setting and the method. In section 4 , we briefly describe three project stories that were written as an autoethnography and discuss our findings. Section 5 concludes our research and provides directions for future research in this area.

\section{Prior work}

We discuss prior work in three major areas of our research, namely the gaps in HCISE integration, autoethnography in organisational context, and HCI effort estimation. 


\subsection{Gaps in HCI and SE}

HCI researchers have been extensively advocating inclusion of HCI practices in SE for more than three decades. Quantitative studies have shown that the better integration of $\mathrm{HCI}$ activities in SE processes leads to software that aligns better with its goals [9].

There have been attempts to integrate specific HCI methods [10] [11] [12] [13] and process models [14] [15] in software development with reasonable success. HCI practitioners have also achieved some success in implementing various User eXperience (UX) team models in software development organisations [16] [17]. Few qualitative case studies and first-person accounts of HCI practitioners through 'action research' of software projects [18] [19] have highlighted practical challenges in HCI-SE integration. And yet, studies do not show encouraging reports on the entrenchment of $\mathrm{HCI}$ in SE, which is evident from the works of some HCI practitioners who have even proposed workarounds and shortcuts to handle these challenges [20] [21] [22] [23] with the view that 'something is better than nothing'.

A review of SE 'textbooks' reveals that any research in the area of HCI-SE integration has not permeated sufficiently in these books, and a clear-cut gap exists especially in the 'requirements' chapters [24] [25] [26]. However, considerable amount of scholarly research in SE discusses various methods to integrate HCI practices in SE. Text on requirements engineering as early as 1993 suggests the use of ethnographic techniques to inform software requirements [27].

In the next three paragraphs, we list the major obstacles in the integration of HCI practices in SE in the industry practice that are identified and summarized in three separate works [9] [18] [28]:

HCI practitioners are involved too late in the project and HCI activities are often included as an afterthought. SE managers think that their project cannot afford to spend time and efforts on usability. There is an overall reluctance to adopt HCI practices, and HCI practitioners are regarded as "nuisance", who get in the way of people who "really deliver" the project. Wherever HCI activities are included, they are not allotted sufficient time in the software development project plan. HCI practitioners are rarely involved in the requirements engineering stage, and software requirements are often over-specified by way of a description of the user interface rather than users' tasks or goals. Usability is considered and documented as a Non-Functional Requirement (NFR) in a software product. Porting projects, i.e. projects undertaken to 'port' the software to a newer technology platform, provide minimal input to HCI practitioners, as the requirements are simply copied from previous versions of the software. Usability is viewed as a 'window dressing' discipline, with focus only on style guides.

SEs and HCI practitioners do not share the same culture or perspective and do not understand the respective constraints under which each group has to operate. There seems to be a significant lack of communication between SEs and HCI practitioners, 
in spite of working together on the same project and from the same location. SEs and HCI practitioners use different names for similar activities and artefacts, often resulting in duplication of efforts and deliverables.

HCI techniques are relatively unknown, and are inaccessible to small and medium sized software development teams. Quite often SEs with little or no training in HCI are directly responsible for practicing and applying HCI methods in their organisations, making crucial HCI decisions without the benefit of usability knowledge. HCI activities do not get sufficient process support from software development organisations. Since inclusion of $\mathrm{HCI}$ activities is not a process improvement, but as a paradigm shift, organisational inertia inhibits the inclusion of HCI methods in their processes. Although HCI practitioners provide several iterations of design solutions, crucial design decisions are often taken by the client representatives.

Though the above gaps are qualitative in nature, most of these tend to describe the effect or the end result, and not the root cause of these gaps. Perhaps these researchers were not looking for the root causes, but intended to implement some specific HCI methods in an SE project, or the research methods that they were using to identify the root causes were not suitable.

One of the gaps that has not been investigated sufficiently, and that we believe has a significant early impact on the success of HCI activities in a project, is that of effort estimation. This is of particular importance in the context of outsourced software development projects.

\subsection{Autoethnography}

Since our research is directed to the practical and sociological aspects of software development, we use ethnographic studies of software engineering community and its practices as an exploratory method to identify the root causes behind the aforementioned gaps in $\mathrm{HCI}$ and SE.

Further, given that the first author of this paper works in a mainstream IT company in India, we choose autoethnography as a method to generate deeper insights. This company provides IT solutions to hundreds of customers across the world. This author has access to several projects in this company and hence enjoys a professional advantage for such a study.

Ethnography is a branch of anthropology dealing with scientific description of cultural phenomena through observation, participation and interpretation [29]. Ethnography is derived from Greek 'ethnos', meaning folk or people of the same race who share a distinctive culture, and 'grapho' meaning 'to write'. The word ethnography refers to both, the method and the result, the study and systematic recording of human cultures, and also a descriptive work produced from such research [30]. 
Ethnographic research methods include conversations, interviews and participant observation. While the first two are indistinguishable from other types of fieldwork, participant observation is a characteristic feature of ethnography. Diverse research techniques are employed here, including analysis of spoken discourses or narratives, collecting and interpreting artifacts, collecting oral and life history material. As a result, ethnographic work involves 'thick descriptions' of a cultural phenomena [31].

Participant observation is the core means of ethnographers to understand the world views of the culture of the researched community by not merely making observations, but also by participating. The term 'Participant Observation' consists of two conflicting or oxymoronic terms [32]. Participation indicates joining the activities, sharing experiences and emotions, contributing to discussions, and taking part in the very interactions that make up a social life. Observation implies an outsider watching and listening, not always taking part, recording the goings-on of the field and gathering other form of material evidence. In short, it entails trying to maintain some sort of dispassionate scientific objectivity. The tension between the two can be difficult to manage in practice. Clifford Geertz describes the participant observer's predicament as "a question of living multiplex life: sailing at once in several seas" [33].

Ethnography is an exploratory research. We use ethnography when we are not sure what research questions or even the categories of questions that we should ask. Focus and research questions emerge and evolve as the researcher learns about the studied practice. In organisational ethnography, it can be used as a comparative method - to make comparisons between the official and unofficial, or what should happen vs. what actually happens, and a comparison of formal vs. informal practices. For organisational ethnography, "the real voyage of discovery begins not with visiting new places but in seeing familiar landscapes with new eyes" [34].

When the ethnographer becomes a part of the people under study, she gains what is called the 'emic' perspective, which, as the insiders' point of view, is the heart of ethnographic research. She attempts to understand and accurately describe situations and behaviour, not objective reality [35]. When she stands back and tries to make sense of the observations, she acquires the 'etic' perspective, or the outsider's point of view, typically from the social scientific perspective of reality, or in the context of organisational ethnography, that of 'what should be'. The etic perspective has also been successfully achieved through collaboration with an academic researcher in organisational ethnography [36]. In software engineering organisations, ethnography has been the key method to inform the design of software for Computer Supported Collaborative Work (CSCW), as because of its unique ability to uncover the complexities of the social aspect of collaboration [37].

Ethnographic reflexivity occurs when the ethnographer looks at the methods and the ways of interpreting that she is applying while doing her field study. She needs to pay careful attention to how her own thinking, or maybe methods, is distorting the sociological phenomenon or biasing it [38]. 
Autoethnography is a specialised version of ethnography. It is a combination of autobiographical ethnography that interjects personal experience with field research, native anthropology where subjects study their own group, and ethnic autobiography that involves personal narratives [39]. As in case of ethnography, autoethnography is both a method and the product of that method. In autoethnography, the researcher "possesses the qualities of permanent self-identification through full internal membership of the researched community" [40], and hence it becomes most relevant in our research context. This method also suits the motivation of this research, since it not only invokes the autoethnographer to awaken to, respect, and make contact with her own questions and problems [41] but also to search for knowledge that can potentially bring about positive changes in the researcher's life [42].

Autoethnographic fieldwork relies on the researcher's personal documentation, including diaries, written communications and personal notes to capture the emotional and subjective aspects of the research. It is, of course, not practical to remember and make note of everything that happens in the researcher's daily life, and autoethnographers can write about important and remembered moments perceived to have significantly impacted the trajectory of subsequent events [42].

This study attempts to gain first-hand insights into the HCI practices in offshore software development from a social perspective, to gain comprehensive understanding of how HCI work is organized in SE processes and its implications to HCI as a profession. It throws light on the social aspects of how HCI activities are situated in the offshore SE context, and how they contribute to the effort estimation and scheduling of HCI activities in offshore software development.

Software development in the India takes place in complex environments that are geographically and culturally distributed, and involve people from various disciplines and cultures. Autoethnography facilitates a detailed account of the practice and situated activity of such a multifaceted environment. Since ethnography is not only about documenting facts, but also about interpretation of those facts, it allows us to view the happenings from a different lens. As a method, it has the unique ability to highlight the local rationalities of people's action and behaviour in the given context. As an exploratory method with constructivist approach, ethnography is most beneficial in under-researched areas. It is therefore relevant to use autoethnography in an attempt to understand the practical and sociological aspects of integration of HCI activities in SE.

\subsection{HCI Effort Estimation}

Per our understanding, no prior research has been done in the area of effort estimation of HCI activities in the outsourced software industry context. Perhaps this is because literature tends to be dominated by either university researchers or by independent HCI consultants, rather than by HCI practitioners in offshore software development organisations. 


\section{Our Study}

The first author of this paper is a full-time employee of a large IT company in India. She has considerable exposure and experience both in SE practices and HCI methods, which provide her with 'unique adequacy', i.e. indigenous skills and knowledge to help her in making sense of the goings-on in the software development community, thus bringing in the emic. The second author is a full-time faculty member of a university, and though he has had interactions with the industry, he is very much an outsider to the industry. There are at least two levels of etic in our work. Firstly, both authors come from an HCI and design discipline which, in a sense, is still considered as 'outsider' to the mainstream software development work. Secondly, the second author is a further 'outsider' to the organisation being studied.

The first author described the project stories as they happened in real life. Given her background, she at times did not question everything and took many events and happenings for granted. This is where the role of the second author came in wherein he questioned the current practices and showed them in a different light. Thus this research is a result of a co-produced autoethnography, where the autoethnographer collaborated with an academician to add richness to the interpretation.

\subsection{Background}

The company in question deals with IT solutions for 540 customers from 46 countries. The company follows an organisational structure of Business Units (BUs) based on the geographical location of the client. Each BU is treated as a separate entity with their own business targets, and contains several Independent software Delivery Units (IDUs) that are supported by Non-Delivery Units (NDUs) that consist of pre-sales and capability development practices.

The user experience practice in this company is called the User Experience Group (UXG) that employs about 100 people. This group is operational since year 2000 and is currently distributed in three BUs. The author of this paper heads the UXG practice of North America geography (UXG-NA), which currently consists of 30 team members (Fig.1). Of these, 17 are HCI practitioners and visual designers with undergraduate and graduate level qualifications in visual communication, interaction design, human factors engineering, computer science and graphic design, with experience ranging from 5 to 12 years in HCI. Several team members have additional certifications in usability, $\mathrm{HCI}$ and project management. The remaining team of 13 works in the UI development area with skills in UI technologies such as HTML/ CSS, Javscript, JQuery, Liferay, Flex. These developers are also sensitized towards HCI to an extent that they can interpret interactive prototypes and visual designs comfortably without the need for detailed design specifications. UXG members are geographically distributed in three cities in India and three cities in the USA. UXG-NA is a part of an NDU of a BU that deals with clients from USA and Canada. 


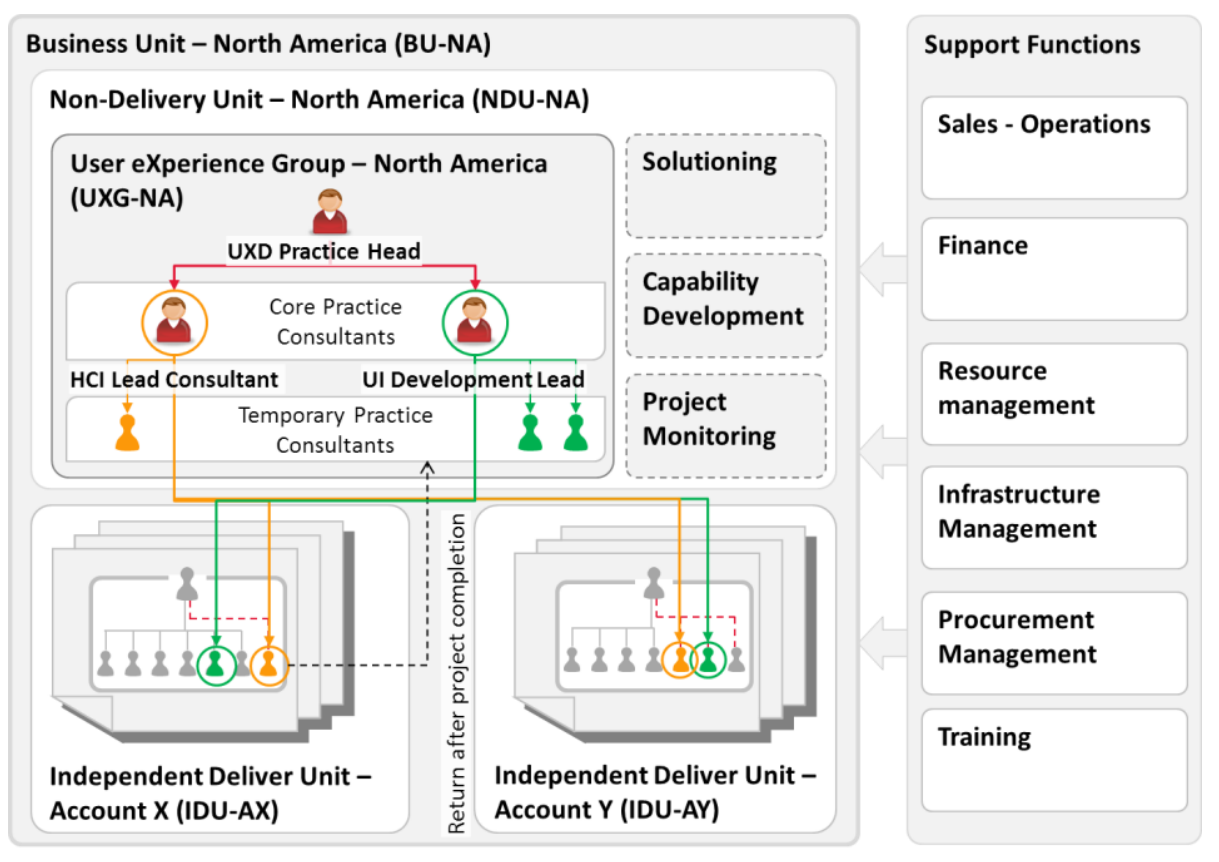

Fig. 1. Organisational structure showing placement of UXG team

UXG is involved in HCI design for various types of projects such as enterprise applications, customer self-service portals and customer support applications. UXG is also involved in pre-sales activities including preparing project proposals, effort estimation for the UX activities of a proposed project, and supporting proofs-of-concept (POCs). In 2013, the UXG-NA team has worked on over 50 projects and POCs. The UXG group and delivery team jointly prepare effort estimates for each project. Finance team quotes a 'dollar figure' to clients based on the effort estimates and the Sales Operations group deals with invoicing the clients.

Once a project is confirmed, an HCI practitioner is assigned to that project in a matrixed project organisation structure. Their direct reporting remains within UXG, but are assigned to work with the project manager as a part of the project team. If more than one person from UXG is assigned to the project, one of them is designated a UXG lead.

When they are not directly assigned to a client project, UXG members are assigned to a central 'practice' project in NDU, where they work on capability development and presales activities. A core team of HCI practitioners as lead consultants are a part of this practice project. These lead consultants from mentor and review the work of the HCI practitioners who are assigned to confirmed projects. 


\subsection{Method}

The first author has been playing the role of an HCI practitioner, mentor and manager for UXG-NA for the past three years. She has been closely involved in the UXG projects from the proposal stages to the closure of UXG participation in the project. While working with her team on these projects, she has encountered several of the known gaps with HCI-SE integration that were discussed in the prior works section. It was felt that if she was to take steps to improve HCI-SE integration, merely identifying the existence and describing the nature of these gaps was not enough. It was necessary to seek a deeper understanding of the root causes of these issues. It was decided to use autoethnographic methods to understand the sociological and practical aspects underlying the causes of these gaps.

The first author started applying autoethnography techniques to her fieldwork and wrote a daily diary and several 'project stories' using her project notes, email communications, meeting notes, project documents, and organisational process documents. This paper refers to the project stories of three projects written between the five months of August 2013 and December 2013, which we believe represent three typical project engagement types in the Indian IT industry. The stories contained thick descriptions of the goings-on at the workplace, in relation to the project, including the autoethnographer's own thought process, perceptions and emotions, and interpretations of actions of her colleagues. Since Project B and C were already in progress when she started writing the diary, the initial parts of these stories were based on retrospective account of the events based on earlier emails, project notes, and the autoethnographer's memory.

These stories were further analysed by making sense of the data by bringing in the academic perspective into what really happened, using interpretations from previous works, which refers to the essential ethnographic technique of 'emic interpreted by the etic'. The original project stories are detailed descriptions of the way the project progressed, the organisation structure and roles of the project team, excerpts from communications, quotes from team meetings, excerpts from project artefacts, etc. that the autoethnographer perceived as 'what matters for HCI'. Further, the stories were written by the first author in the role of an autoethnographer, but the analysis was conducted by collaborating with the second author, whose academic background helped with the etic perspective in identifying issues and their causes in the light that were not viewed by the research community before. For the sake of this research paper, we abridged the project stories only in the context of our findings related to HCI effort estimation.

\section{$4 \quad$ Findings and Discussion}

To provide context to our findings we present a short description of the three representative project stories written as a part of the research. We further discuss our findings from these project stories in the way they influenced the project outcome. 


\subsection{Project stories}

The projects represent three typical types of commercial terms of engagement with customers in outsourced software development; the first is Fixed Price model, the second, a Mixed model, and the third, Time \& Material model. These stories were written in a narrative format using a first person narrative ' $I$ ' in case of autoethnographer's individual actions and thoughts, and 'we' in case an additional UXG member was involved.

The Fixed Price (FP) model is typically used where the scope of work is clearly defined, making it possible to estimate the efforts required fairly accurately and thus charge the customer with an agreed fee for a well-defined scope of work. The Time \& Material (T\&M) billing is done using an agreed hourly rate based on consultants' time spent and other costs such as software and infrastructure. A Mixed model is used when consulting services are required for defining the scope of project work which, once defined, allows a more precise estimation of the efforts for subsequent design, development and testing activities. In this model, T\&M billing is adopted for initial activities related to identifying scope and requirements, and a fixed fee is charged for the subsequent clearly defined scope of work. The FP and Mixed models are typically used when the vendor company is providing IT consulting services to the customer. However, when conducting business with IT divisions of large customer organisations having their own IT teams with their own program and project management, a T\&M model is traditionally used for 'staff augmentation'. This is essentially 'filling up' vacant job positions within the customer organisations on contract basis, where monthly invoices are sent to the customer for each position as per T\&M billing rates agreed upon in a Master Service Agreement (MSA) signed with the customer. The customer 'sponsor' often sends 'job descriptions', interviews candidates for these positions and issues work orders for start and end dates of the selected employees. These employees then either move to customer locations overseas or work from the customer's Offshore Development Centres (ODCs) which are secure offices connected to the customer's network but located in India within the IT company's premises.

Project A: UXG was asked by a senior member of the sales team to provide an FP estimate in about four hours with limited understanding of the scope as "preparing wireframes/ HTML pages of an application consisting of 25 pages" and to refer a requirements document prepared earlier by the presales team. This project arrived around the time the authors decided to write project stories as a part of autoethnographic research, and saw it as an opportunity to write the story as the project progressed. Hence this story does not contain any retrospective accounts. The said requirements document was an over-specification of the User Interface (UI) to an extent of a detailed description of the proposed UI, including description of links and controls to be provided on each page. We (UXG) interpreted the document from 'user tasks' perspective, made assumptions to arrive at a count and complexity of the probable tasks, and applied our standard estimation template to quote our efforts for HCI activities. Since we (UXG) had not worked with this customer before, we were urged by the sales leads to provide "conservative" estimates in order to get a "foot-in-the- 
door" to gain entry with this customer. The software development activities had already started, and since there was not enough time to invest in user studies, we had to completely rule out that activity from our estimates. Once the project started, the 'onsite' delivery lead asked us to "produce screens" without giving us any input on users' goals and task flows. Since the development estimates (also FP) were based on the said requirements document, we were asked not to digress from it and to refrain from proposing any design solution that would change the development effort. The software development team was required to review our design to ensure that we do not propose any design element that calls for a deviation in the development effort. However, the development team was occupied with their work, and our designs were taken to the customer representative for review without the development team's feedback on possible development scope changes. Due to limited initial input, our conceptual design was a far cry from the expectations of the customer representative, who had to spend time with us to explain to us the user goals and tasks. After ten days of deliberation, reviews and UI suggestions from the customer representative, our wireframe prototypes took shape, seemed to meet customer expectations and were signed off by the customer representative.

The wireframes were then passed on to the development team for implementation. That is when they realised that the development efforts would increase significantly if they were to use our wireframes. They raised a change request (CR) and quoted additional efforts to the customer for the changes. The customer was unable to procure additional funds due to lack of business justification, and consequently, we were asked to re-design the UI to match its description in the original requirements document. Our value-add was thus mostly limited to visual design as per the customer's brand guidelines. The delivery team perceived the resulting wastage of time and duplication of efforts as our fault, since apparently visual design was what was expected from us in the first place. Eventually, though a better design was produced and approved by the client representative, it was never implemented. Further, the project timeline was affected, and we left a bad impression with the customer as well as the delivery team.

Project B: UXG team of two HCI practitioners was already working on this project from the customer location in the USA for a year at the time we wrote this project story. The commercial engagement with the customer was on a Mixed Model basis. This was a greenfield project, i.e. a project which is independent of any constraints from prior software applications in terms of inherited technology, processes or functionalities. Since the scope was unknown at the requirements stage, the customer was charged on T\&M basis for the HCI design activity. Subsequent visual design and development work was done on an FP basis. The customer had their own HCI team working on a part of this project. When additional scope was anticipated in the next releases, the delivery team proposed addition of UXG team members to work from offshore locations on T\&M basis for working on the detailed design. Also, the customer's internal HCI team members were discontinued from this project for various political reasons, creating the need to add UXG team members on this project. Thus, a team of three additional HCI practitioners were added to the existing team for the new scope of work at the time we wrote this project story. 
As per the client's information security mandate, these offshore HCI team members were required to work from the customer's ODC, having very strict security policies. The security controls included very limited internet access, restrictions on carrying books or mobile phones inside the ODC, and mailbox audits for possible violations.

As things turned out, the additional work that was anticipated was not enough to fully engage these three additional team members. The security policy restricted the installation of any software on the local machines and the offshore UXG team was required to work with software like Photoshop and Axure installed on remote desktops, resulting in severe loss of productivity of the offshore team. Mechanisms for file sharing between onsite and offshore team also proved to be tedious. The result was that the onsite team ended up doing most of the design and prototyping work and the offshore team was left with little or no work. Project delivery heads were unwilling to release them from the project, as it would mean loss of revenue in T\&M mode. They also anticipated additional work in the near future, and were unwilling to let go of the team who were already familiar with the project, for fear that they may not be able to get the same team when additional work comes in. Eventually, one HCI designer left the organisation as he was left idle for too long. The customer also became aware of the lack of offshore productivity resulting in removal of one additional HCI practitioner.

Project C: Two HCI practitioners were working on this project for over a year on this program when we wrote this story. The customer belongs to the IT department within the client organisation. This was a porting project, where the new software was to have only a few additional features and functionality from a legacy application. All the team members involved in this project, including the program manager, were on staff augmentation (T\&M) mode. A customer manager coordinated the activity of defining minor additional requirements and the scope for the next release. He planned the start date for each release, when the HCI activities started on the same date as development activity.

As per this delivery schedule, we allocated two HCI practitioners who started at the same time as the development and UI integration team. For the development team, however, the starting point for their work was the UI code, which was in fact the final deliverable of the UXG team. Since the developers' time was billed to the customer, they were required to produce evidence of their work and hence they demanded the UI code on day one. The UXG team was hard pressed on time to deliver the design and UI code in the first part of the release cycle. On the other hand, once the UI was delivered, the UXG team was left with very little support work till the end of the cycle. Conversely, the development team, having lost valuable time in the beginning of the phase, was overloaded at the end.

We recommended to the customer manager to stagger the start dates of UXG team and development team. However, from his perspective, each development cycle takes into account an approved budget for an approved scope of work, and he found it practically difficult to implement our suggestions. Therefore, this schedule continued for two cycles regardless of our recommendation. From the third cycle onwards, the 
UXG team members started to proactively utilize their idle time at the end of release to conduct HCI activities based on our 'gut feel' about the next logical scope for the fourth release. When scope was finalized for the fourth release, it turned out to be slightly different from our guess, but we were able to effectively use our design work with slight changes in the fourth release, thus getting a buy-in for our recommendation from the customer manager.

From the fourth release onwards, we were able to implement this staggering arrangement, which has now been running smoothly for the next three releases.

\subsection{Discussions}

Effort estimation and schedule of HCI activities in outsourced IT projects has not been discussed within HCI. Our study provided insights into this under-researched area from the perspective of causality.

\section{Layers of negotiations.}

HCI practitioners in outsourced software development companies have to deal with multiple layers of negotiation for their estimates and schedules, starting with sales teams, project delivery heads, development teams and then finally with the client. By the time we reach the client level, the effort estimates are reduced by $10-20 \%$ of the initial estimate. Our negotiations are not just limited to efforts, but also extend to the justification of proposed HCI activities affecting development timelines.

\section{Foot-in-the-door becomes foot-in-the-mouth .}

With "conservative" estimates for efforts as well as timelines, we are forced to cut down some crucial user-centred design activities, not only affecting the quality of our design output but also creating undesirable precedents for future engagements.

While trying to gain entry with a new customer, we tend to spend crucial time in the initial design stage in getting familiarized with the new organisation and new domain. Already pressured with a compressed estimate, this unavoidable activity is seen as wastage of time due to HCI practitioners' lack of domain expertise, creating a bad impression with the customer.

In case of Project A, the customer requested UXG involvement as an afterthought in an attempt to get a better UI which was perceived as a business opportunity by our sales team. However, since these events took place after development scope was finalized, slight deviation from the scope meant a Change Request in a Fixed Price project, triggering a change management process. This change request did not get budgetary approval due to insufficient business justification for the change, as the scope of work had never changed in the first place. 
Effectively, the project did not benefit much from the HCI expertise, created a bad precedent and bad impression, and also failed to meet the original objective of getting foot-in-the-door with a new customer.

\section{Over estimation leads to under performance and inefficiency}

Whereas in foot-in-the-door Fixed Price projects there is pressure on the UXG to minimise efforts put in the project and still make a good impression on the client, the pressure is in the opposite direction in case of a T\&M project. Particularly for greenfield projects, with little clarity on project scope, project management literature suggests that it is safe for both the parties to sign in a T\&M contract [43]. In case of large programs, as seen in Project B, the project management tends to over-estimate the human resources requirement, with a view to generating maximum revenue from the engagement.

This, coupled with an offshore software development scenario, the information security limitations, and the work environment in certain customer ODCs makes it difficult to utilize the full potential of offshore HCI practitioners, leading to inefficiency, employee dissatisfaction and attrition.

\section{Relay race is better teamwork than race against time.}

Since HCI efforts are perceived as development activity, or at best, low level design activity, our project schedule is planned concurrently with the UI integration and backend development schedule. As seen in Project $C$, this resulted in unequal work distribution for the entire team during the development phase. The resulting work pressures amplified the lack of cooperation and co-ordination between software developers and HCI practitioners. When software development is iterative and the project plans to release multiple cycles, there is an opportunity to stagger the HCI and software development work. This approach is also the one used for HCI activities in agile software development projects, as suggested in literature on integration of HCI in Agile SE process [44] [45]. Staggering of start date for HCI activities in a project resulted in equal distribution of workload for both UXG and the software development teams leading to optimised resource utilisation. Instead of running a race against time, relaying design output in a staggered manner resulted in improved co-ordination amongst two teams, better work distribution, and effectively better output. However, current practices of organising such projects around features, makes it difficult to stagger the work in this manner. Though a desirable change was brought about through proactive and long-term involvement of the HCI practitioners in the particular case of Project $\mathrm{C}$, such practices need generalisation and mainstreaming. 


\section{Conclusion}

Our research led to a better understanding of effort estimation and scheduling of activities in the offshore software development context. We found that HCI practitioners have to continually deal with several layers of negotiations for estimating and scheduling HCI activities. In case of FP estimations, especially the foot-in-the-door mode led to cost cutting, insufficient $\mathrm{HCI}$ activities, time pressures, poor impressions, and poor quality of design output. The T\&M estimation, on the other hand, allows sufficient time to conduct HCI activities leading to better quality of design output, but failed in terms of resource utilization for certain customers. In another case, better management and planning HCI in software development activities improved work distribution, cooperation and co-ordination amongst HCI and SE community working in T\&M mode.

The issues related to HCI-SE integration literature in the context of industry practices have referred to the challenges that are faced by the HCI practitioners. Our findings provide deeper insights to the social situations and situated actions of individuals in the setting that led to some of these challenges for HCI effort estimation.

We found benefits in the use of autoethnography as a research method in providing deeper understanding of HCI projects in offshore SE environment. Previously, when the first author worked without the current research angle, she took some actions as 'given', as something 'that was always done' without giving them much thought. Collaborating with the academic co-author for analysing the project stories helped in comparing the 'what happened' against 'what should have happened' which she would have otherwise missed as 'what typically happens'. When she started thinking about her own actions as a part of the 'observing' in autoethnography, she may have affected her thoughts and actions reflexively. While this position is similar to that of a reflective practitioner [46], our work brings in the ethnographic element of the etic perspective to inform the reflections of the emic aspects.

In order to understand the perspectives and interpret the actions of others around her in the researched field, the first author gained renewed empathy with other disciplines, including software development teams, the sales teams (in Project A) as well as the client manager in (Project C).

However, the ethnographer found it extremely difficult to observe emotions, reactions and level of involvement in distributed teams, since a majority of communication takes place over teleconference and emails. We think that engaging in regular informal conversations outside of work related communications with co-workers, both from HCI and SE areas, can help in overcoming this limitation in future work. This level of insights into the rationale of individuals behind situated actions can allow deeper understanding of the causality of issues. 
The use of autoethnography in outsourced software development industry from an HCI perspective can be extended towards identifying the root causes for several other gaps found prevalent in HCI-SE integration, the most pressing issues being the absence of HCI practices and practitioners in requirements engineering, lack of support from organisational processes, and perception of HCI activities as 'skin deep'.

\section{Bibliography}

[1] A. Seffah, M. C. Desmarais and E. Metzker, "HCI, usability and software engineering integration: present and future," Springer, 2005, pp. 37--57.

[2] A. Abran and P. Bourque, SWEBOK: Guide to the software engineering Body of Knowledge, IEEE Computer Society, 2004.

[3] J. Doerr and A. L. Opdahl, "Foundation for Software Quality".

[4] NASSCOM, "The IT-BPM Sector in India: Strategic Review 2013," 2013.

[5] R. A. Majid, N. L. M. Noor and W. A. W. Adnan, "Strengthening the HCI Approaches in the Software Development Process," in Proceedings of World Academy of Science, Engineering and Technology, 2012.

[6] B. Jerome and R. Kazman, "Surveying the Solitudes: An Investigation into the Relationships between Human Computer Interaction and Software Engineering in Practice," in Human-Centered Software Engineering - Integrating Usability in the Software Development Lifecycle, A. Seffah, J. Gulliksen and M. C. Desmarais, Eds., Springer, 2005.

[7] K. Rönkkö, Making methods work in software engineering: Method deploymentas a social achievement, 2005.

[8] K. Rönkkö, J. Winter and M. Hellman, "Reporting user experience through usability within the telecommunications industry," in Proceedings of the 2008 international workshop on Cooperative and human aspects of software engineering, 2008.

[9] A. N. Joshi, "Integration of Human-Computer Interaction Activities in Software Engineering for Usability Goals Achievement," IIT Bombay, 2011.

[10] H. Fischer, "Integrating usability engineering in the software development lifecycle based on international standards," in Proceedings of the 4th ACM SIGCHI symposium on Engineering interactive computing systems, 2012.

[11] S. Dupuy-Chessa, N. Mandran, G. Godet-Bar and D. Rieu, "A Case Study for Improving a Collaborative Design Process," Springer, 2011, pp. 97--101.

[12] C. Leonardi, L. Sabatucci, A. Susi and M. Zancanaro, "Design as intercultural dialogue: coupling human-centered design with requirement engineering methods," Springer, 2011, pp. 485--502.

[13] B. Al-Ani, E. Trainer, R. Ripley, A. Sarma, A. Van Der Hoek and D. Redmiles, "Continuous coordination within the context of cooperative and human aspects 
of software engineering," in Proceedings of the 2008 international workshop on Cooperative and human aspects of software engineering, 2008.

[14] T. Memmel, F. Gundelsweiler and H. Reiterer, "CRUISER: A Cross-Discipline User Interface and Software Enginee ring Lifecycle," Springer, 2007, pp. 174-183.

[15] K. S. Sousa and E. Furtado, "An approach to integrate HCI and SE in requirements engineering," in Proc. of Interact'2003 Workshop on Closing the Gaps: Software Engineering and Human-Computer Interaction, 2003.

[16] U. Gajendar and C. Johnson, "The inmates are still running the asylum: How to share a design vision with engineers," Springer, 2011, pp. 276--282.

[17] J. E. Nieters, S. Ivaturi and G. Dworman, "The internal consultancy model for strategic UXD relevance," in CHI'07 extended abstracts on Human factors in computing systems, 2007.

[18] J. Patton, "Hitting the target: adding interaction design to agile software development," in OOPSLA 2002 Practitioners Reports, 2002.

[19] J. Gulliksen, B. Göransson, I. Boivie, S. Blomkvist, J. Persson and Å. Cajander, "Key principles for user-centred systems design," Behaviour and Information Technology, vol. 22, no. 6, pp. 397--409, 2003.

[20] C. Bowles and J. Box, Undercover User Experience Design, Pearson Education, 2010.

[21] S. Krug, Rocket surgery made easy: The do-it-yourself guide to finding and fixing usability problems, New Riders, 2009.

[22] N. Khalayli, S. Nyhus, K. Hamnes and T. Terum, "Persona based rapid usability kick-off," in CHI'07 extended abstracts on Human factors in computing systems, 2007.

[23] L.-C. Lin and W. O. Lee, "UI toolkit for non-designers in the enterprise applications industry," in CHI'07 extended abstracts on Human factors in computing systems, 2007.

[24] R. S. Pressman, Software Engineering - A Practitioner's Approach, 7th.ed. ed., McGraw-Hill, 2010.

[25] I. Sommerville, Software Engineering. International computer science series, Addison Wesley, 2004.

[26] R. Y. Lee, Software Engineering- A Hands-On Approach, Atlantis Press, 2013.

[27] I. Sommerville, T. Rodden, P. Sawyer, R. Bentley and M. Twidale, "Integrating ethnography into the requirements engineering process," in Requirements Engineering, 1993., Proceedings of IEEE International Symposium on, 1993.

[28] A. Seffah, J. Gulliksen and M. C. Desmarais, Human-Centered Software Engineering-Integrating Usability in the Software Development Lifecycle, vol. 8, Springer, 2005.

[29] S. C. Galman, Shane, The Lone Ethnographer - A Beginner's Guide to Ethnography, AltaMira Press, 2007.

[30] P. Atkinson, A. Coffey, S. Delamont, J. Lofland and L. Lofland, Eds., Handbook 
of Ethnography, London: Sage Publications Ltd., 2001.

[31] R. Madden, Being Ethnographic - A Guide to the Theory and Practice of Ethnography, Sage, 2010.

[32] K. O'Reilly, Key Concepts in Ethnography, Sage, 2009.

[33] C. Geertz, Works and Lives: The Anthropologist as Author, Stanford University Press, 1988.

[34] S. Ybema, D. Yanow, H. Wels and F. Kamsteeg, Organizational Ethnography Studying the Complexity of Everyday Life, Sage Publications, 2009.

[35] M. Hammersley and P. Atkinson, Ethnography: Principles in practice, Routledge, 2007.

[36] S. Kempster, J. Stewart and K. W. Parry, "Exploring co-produced autoethnography," Business papers, p. 112, 2008.

[37] D. Shapiro, "The limits of ethnography: combining social sciences for CSCW," in Proceedings of the 1994 ACM conference on Computer supported cooperative work, 1994.

[38] C. A. Davies, Reflexive ethnography: A guide to researching selves and others, Routledge, 2008.

[39] D. E. Reed-Danahay, Auto/ethnography, Berg New York, 1997.

[40] D. M. Hayano, "Auto-ethnography: Paradigms, problems, and prospects," Human organization, vol. 38, no. 1, pp. 99--104, 1979.

[41] S. Wall, "An autoethnography on learning about autoethnography," International Journal of Qualitative Methods, vol. 5, no. 2, pp. 146--160, 2008.

[42] C. Ellis, T. E. Adams and A. P. Bochner, "Autoethnography: an overview," Historical Social Research/Historische Sozialforschung, pp. 273--290, 2011.

[43] A Guide to the project Management Body of Knowledge (PMBoK Guide), 5th Ed. ed., Pennsylvania USA: Project Management Institute, 2013.

[44] A. Joshi, N. Sarda and S. Tripathi, "Measuring effectiveness of HCI integration in software development processes," Journal of systems and software, vol. 83, no. 11, pp. 2045--2058, 2010.

[45] H. Beyer, K. Holtzblatt and L. Baker, "An agile customer-centered method: rapid contextual design," Springer, 2004, pp. 50--59.

[46] D. A. Schon, "The reflective practitioner: How professionals think in action," 1984.

[47] H. Garfinkel and H. Sacks, On Formal Structures of Practical Actions In Theoretical Sociology: Perspectives and Developments, J. McKinney and E. Tiryakian, Eds., New York: Appleton-Century-Crofts, 1970. 